\title{
RELATING A SPECTRAL INDEX FROM MODIS AND TOWER-BASED MEASUREMENTS TO ECOSYSTEM LIGHT USE EFFICIENCY FOR A FLUXNET-CANADA CONIFEROUS FOREST
}

\author{
Elizabeth M. Middleton ${ }^{a}$, Yen-Ben Cheng ${ }^{a}$, Thomas Hilker ${ }^{b}, K^{2}$ arl F. Huemmrich ${ }^{c}$, T.Andrew Black ${ }^{d}$, \\ Praveena Krishnan, and Nicholas.C. Coops ${ }^{\mathrm{c}}$ \\ ${ }^{a}$ National Aeronautics and Space Administration, Goddard Space Flight Center, Greenbelt, MD, USA \\ ${ }^{b}$ Faculty of Forest Resource Management, University of British Columbia, Vancouver, British Columbia, Canada \\ ${ }^{\mathrm{c} J o i n t}$ Center for Earth System Science, University of Maryland Baltimore County, Baltimore, MD, USA \\ ${ }^{\mathrm{d}}$ Faculty of Land and Food Systems, University of British Columbia, Vancouver, British Columbia, Canada \\ National Oceanic Atmospheric Administration, Oak Ridge, TN, USA
}

\begin{abstract}
As part of the North American Carbon Program effort to quantify the terrestrial carbon budget of North America, we have been examining the possibility of retrieving ecosystem light use efficiency (LUE, the carbon sequestered per unit photosynthetically active radiation) directly from satellite observations. Our novel approach has been to compare LUE derived from tower fluxes with LUE estimated using spectral indices computed from MODIS satellite observations over forests in the Fluxnet-Canada Research Network, using the MODIS narrow ocean bands acquired over land. We matched carbon flux data collected around the time of the MODIS mid-day overpass for over one hundred relatively clear days in five years (2001-2006) from a mature Douglas fir forest in British Columbia. We also examined hyperspectral reflectance data collected diurnally from the tower in conjunction with the eddy correlation fluxes and meteorological measurements made throughout the 2006 growing season at this site. The tower-based flux data provided an opportunity to examine diurnal and seasonal LUE processes and their relationship to spectral indices at the scale of the forest stand. We evaluated LUE in conjunction with the Photochemical Reflectance Index (PRI), a normalized difference spectral index that uses 531 $\mathrm{nm}$ and a reference band to capture responses to high light induced stress afforded by the xanthophyll cycle. Canopy structure information, retrieved from airborne laser scanning radar (LiDAR) observations, was used to partition the forest canopy into sunlit and shaded fractions throughout the day, on numerous days during 2006. At each observation period throughout a day, the PRI was examined for the sunlit, shaded, and intermediate canopy segments defined by their instantaneous position relative to the solar principal plane (SPP). The sunlit sector was associated with the illumination "hotspot" (the reflectance backscatter maximum), the shaded sector with the "cold or dark spot" (the reflectance forward scatter minimum), while the intermediate, mixed sunlit/shade sector was located in the cross-plane to the SPP. The PRI indices clearly captured the differences in leaf groups, with sunlit foliage exhibiting the lowest values on sunny days throughout the 2006 season. When tower-based canopylevel LUE was recalculated to estimate foliage-based values $\left(L U E_{\text {foliage }}\right)$ for the three foliage groups under their incident light environments, a strong linear relationship for PRI:LUE foliage was demonstrated $\left(0.6 \leq \mathrm{r}^{2} \leq 0.8\right.$, $\mathrm{n}=822, \mathrm{P}<0.0001$ ).

The MODIS data represent relatively large areas when acquired at nadir $\left(\sim 1 \mathrm{~km}^{2}\right)$ or at variable off-nadir view angles $\left(\geq 1 \mathrm{~km}^{2}\right)$ looking forward or aft. Nevertheless, a similar relationship between MODIS PRI and tower-based LUE was obtained from satellite observations $\left(\mathrm{r}^{2}=0.76, \mathrm{n}=105, \mathrm{P}=0.026\right)$ when the azimuth offsets from the SPP for off-nadir observations were considered. At this relatively high latitude of 50 degrees, the MODIS directional observations were offset from the SPP by approximately 50 degrees, but still represented backscatter or forward scatter sectors of the bidirectional reflectance distribution function (BRDF). The backscatter observations sampled the sunlit forest and provided lower PRI values, in general, than the forward
\end{abstract}


scatter observations from the shaded forest. Since the hotspot and darkspot were not typically directly observed, the dynamic range for MODIS PRI was less than that observed in the SPP at the canopy level; therefore, MODIS PRI values were more similar to those observed in situ in the BRDF cross-plane. While not ideal in terms of spatial resolution or optimal viewing configuration, the MODIS observations nevertheless provide a means to monitor forest under stress using narrow spectral band indices and off-nadir observations. This research has stimulated several spin-off studies for remote sensing of LUE, and demonstrates the importance of the connection between ecosystem structure and physiological function. 